\title{
Inhaled Loxapine for the Management of Acute Agitation in Bipolar Disorder and Schizophrenia: Expert Review and Commentary in an Era of Change
}

\author{
Bruno Pacciardi ${ }^{1} \cdot$ Alfredo Calcedo $^{2} \cdot$ Thomas Messer $^{3}$
}

Published online: 5 February 2019

(c) The Author(s) 2019

\begin{abstract}
Agitation is a common and costly phenomenon associated with a number of psychiatric conditions including schizophrenia and bipolar disorder. Early identification and prompt intervention to relieve the symptoms of agitation are essential to avoid symptomatic escalation and emergence of aggressive behaviour. Recent consensus guidelines emphasise the need for noncoercive management strategies to protect the therapeutic alliance between patients and their healthcare providers-an alliance that is critical for the effective management of chronic psychiatric conditions. Rapid symptom relief and de-escalation of agitation are necessary to avoid the costly and traumatic use of coercive techniques of physical restraint and seclusion, which require admission and prolonged hospitalisation. Inhaled loxapine is approved for the treatment of acute agitation in patients with schizophrenia or bipolar disorder. Clinical studies have confirmed the efficacy, rapid onset of action, and safety and tolerability of this agent in the psychiatric emergency and hospital settings. Emerging data have indicated the potential for inhaled loxapine as a self-administered agent for use in the community setting without the direct supervision of a healthcare professional. We discuss the evolving treatment paradigm and the place of inhaled medications for acutely agitated patients both within and outside the emergency and hospital setting.
\end{abstract}

Bruno Pacciardi

bruno.pacciardi@gmail.com

Azienda Ospedaliera Universitaria Pisana, Pisa, Italy

2 Hospital General Universitario Gregorio Marañón, Madrid, Spain

3 Danuvius Klinik GmbH, Pfaffenhofen an Der Ilm, Germany

\section{Key Points}

Agitation is common among patients with schizophrenia or bipolar disorder and its management can be both traumatic and costly when coercive methods, physical restraint and inpatient treatment with prolonged hospitalisation are required.

Early non-physical management of the agitated patient with non-invasive de-escalation techniques, which aim to reduce agitation and distress and avert aggression or violence, protects the therapeutic alliance between patients and their healthcare providers and acknowledges the ethical and legal rights of individual patients.

Inhaled loxapine is approved for the treatment of acute agitation in adults with schizophrenia and bipolar disorder and can be used as part of non-coercive management protocols alongside techniques such as verbal de-escalation for cooperative patients and may be a suitable agent for self-administered treatment of agitation in a community setting from the onset of symptoms. 


\section{Introduction}

Schizophrenia and bipolar disorder affect 23 million and 60 million individuals, respectively, worldwide [1]. Patients with schizophrenia experience psychotic symptoms including distortions of perception, emotion, and thinking including hallucinations and delusions. Patients with bipolar disorder typically experience manic, hypomanic, depressive and mixed affective episodes separated by periods of normal mood $[2,3]$. Both disorders can vary in severity and can impact the patient's quality of life, their interpersonal relationships and their ability to work, and both disorders are associated with an increased risk for suicide [2, 4-6].

Agitation is a component of many psychiatric disorders including schizophrenia and bipolar disorder and can be expressed as increased responsiveness to stimuli, irritability, excitement, physical or verbal aggression (fighting, throwing, verbal outbursts), and non-aggressive behaviours (wandering, pacing, rapid speech, restlessness) [7, 8] (Fig. 1). Agitation is recognised by the American Psychiatric Association as a component of the behavioural characteristics of schizophrenia and as a diagnostic criterion for bipolar disorder [9]. According to the current guidelines issued by the National Association of Psychiatric Intensive Care Units [10], it is part of the more general concept "acute disturbance", together with aggression and violence, in the context of an underlying disease. Severely agitated patients represent a risk to themselves and to their caregivers and healthcare providers.

The treatment paradigm for agitation in patients with schizophrenia or bipolar disorder focuses on early identification and prompt intervention with de-escalation followed by rapid tranquilisation if needed, with pharmacological treatment-ideally fast, consensual and non-invasive-to avoid symptom escalation $[7,11,12]$. In the early stages of management (the pre-rapid tranquilisation phase), 'deescalation' should be an explicitly collaborative process involving a range of verbal and non-verbal interventions. De-escalation aims to reduce agitation and distress and avert aggression or violence. Non-coercive de-escalation techniques are preferred to protect the therapeutic alliance between patients and their healthcare providers as well as to respect the ethical and legal rights of individual patients $[10,12]$. For patients requiring pharmacological intervention, consent and the route of administration are key considerations. Intravenous administration may require physical restraint and should be avoided when possible. Intramuscular (IM) delivery may be acceptable for patients able to consent to such an invasive route of administration. Oral or sublingual delivery may be acceptable for those patients whose agitation is sufficiently mild to allow them to swallow the medication. However, oral administration is associated with a delayed onset of action when both patients and staff remain at risk for the consequences of symptom escalation $[7,12]$. Indeed, the delayed onset of action of oral formulations is recognised as a significant barrier to the effective management of acutely agitated patients and to the establishment and maintenance of the therapeutic alliance between patients and their healthcare providers [13].

Most recently, an inhaled antipsychotic formulation has been developed to reduce the time to the onset of action and is a preferred route of administration [12, 14, 15]. The active agent, loxapine, has been utilised in psychiatric practice for over 40 years [16] and is now available in an inhaled formulation delivered by $\mathrm{OBT}^{\circledR}$ (One-breath-technology) using the Staccato ${ }^{\circledR}$ system. This hand-held, single-use, breathactuated inhaler has been specifically designed to rapidly deliver loxapine as a dry powder into the alveoli of the lungs [17]. This article provides a review of the efficacy and safety profile of inhaled loxapine for the treatment of acute agitation in patients with schizophrenia or bipolar disorder and the place of inhaled medications in the evolving management paradigm for acutely agitated patients.

\section{Burden of Agitation in Schizophrenia and Bipolar Disorder}

Agitation is a common symptom among patients presenting for emergency psychiatric care [8] and, if not recognised and managed promptly and effectively, can escalate to violent behaviour [11]. In USA, an estimated $21 \%$ of psychiatric emergency visits involve patients with agitation [18]. A more recent European study found that during a single week in 2014, 9.4\% of all psychiatric emergency visits to emergency rooms at 27 centres in three countries were for acute agitation [19]. In the inpatient setting, agitation is a common precedent to violence [20]. Agitation-associated violence may be directed towards staff, other patients, visitors or the patients themselves.

\section{Management of Patients with Agitation and Maintenance of the Therapeutic Alliance}

Antipsychotics are the mainstay of therapy for the symptomatic management of schizophrenia [21] and of bipolar disorder [22]. A characteristic feature of both disorders is persistent mood instability [23]. The unpredictability of these disorders means that the needs of such patients extend beyond symptomatic management. Such patients require a holistic approach to care that is underpinned by a strong therapeutic alliance between patients and their caregivers to manage the day-to-day symptoms and support patients in 
adhering to their treatment regimen, enable the recognition of acute symptomatic worsening, including agitation, and to collaboratively intervene when symptoms emerge [22, 24].

Interventions to manage the agitated patient must ensure an achievement of calmness without sedation [10] to keep the patient, the staff and any others safe [12]. In the past, the management of acute agitation has largely consisted of mechanical physical restraint and seclusion. However, it has become increasingly recognised that such an approach as a first response may not be the most appropriate or the most ethical intervention and may compromise any attempt to establish or maintain a therapeutic alliance with the patient $[25,26]$. In Germany, for example, the Federal Constitution Court (Bundesverfassungsgericht) ruled on July 2018 to limit the physical restraint of patients because of the interference with their fundamental right to freedom, especially when the restraint lasts for more than $30 \mathrm{~min}$. This decision will mean that physical restraint in Germany will be restricted to comply with these legal requirements [27].

To support the move away from mechanical physical restraint towards a less coercive and less invasive management strategy in a psychiatric hospital setting, protocols have recently been developed [10, 28]. The former protocol was based on the recent international consensus guidelines [7] that emphasise the need for early identification and environmental and safety management, followed by a step-wise approach moving from verbal de-escalation to pharmacotherapy, with mechanical physical restraint reserved as a last resort [28]. The latter protocol was developed by The British Association for Psychopharmacology and the NAPICLSU in the UK [10] and includes up-to-date recommendations, treatment algorithms with various pharmacological options based on the route of administration and a level of evidence that supports the different options. Both protocols highlight the importance of verbal de-escalation and the relevance of the patient's role in the management of the agitation episode. Verbal de-escalation should always be attempted to reduce the risk for further escalation and establish a therapeutic partnership with the patient for the management of the acute episode. If verbal de-escalation is effective in engaging the patient in a cooperative therapeutic alliance, and at least somewhat effective in relieving or stabilising the symptoms, the need for pharmacological intervention can be considered and the treatment algorithms implemented.

The route of administration (IM, oral/sublingual or inhaled) should, where possible, be chosen by the patient. In an emergency setting, the proposal of a self-administered and non-invasive drug treatment may be an important tool to assess the ability of the patient to cooperate. Oral/sublingual or inhaled routes are preferred for cooperative patients, with IM medications reserved for patients unable to cooperate sufficiently. If verbal de-escalation with or without pharmacological support remains ineffective, then physical restraint can be considered, and the specific algorithm implemented with the aim of administering effective medication and removing the physical restraint in as short a time as possible.

The overarching principles that should form the backbone of any protocol for the acute management of agitation in patients with schizophrenia or bipolar disorder are to: facilitate an early identification of the episode; prioritise verbal de-escalation; avoid coercive measures; establish and maintain a therapeutic collaboration with the patient and include his/her opinion in decisions regarding his/her treatment; and ultimately protect and strengthen the therapeutic alliance between patients and their healthcare providers. The development and implementation of protocols for the management of patients with agitation in the inpatient care setting should be supported by education/training programmes in each centre with accurate medical records and the availability of relevant clinical data for individual patients to facilitate timely treatment decisions based on the individual situation and patient's needs [12].

For agitated patients, in the acute care or hospital setting, it is important to recognise that they should be considered competent to make their own healthcare decisions unless the contrary is proven (Fig. 2). Agitation should be considered a continuum from mild to severe, and the more agitated the patient becomes, the less rational and competent he/she becomes. Severely agitated patients are the least likely to be competent to make decisions about their care while the majority of mildly agitated patients will be able to competently consider and select their preferred therapeutic option. When patients are only partially competent (with escalating or moderate agitation), healthcare providers should seek to maximise their competence through a process of assisted decision making. We, as clinicians, now have a mandate to maximise the remaining decision-making abilities that the patient may have intact [29]. We should initiate a dialogue with the patient and explain their risk of worsening and the recommend pharmacological treatment options including the route of administration (oral, inhaled or IM). The patient should be informed that they may reject a pharmacological option at this stage, but if their agitation worsens then a pharmacological intervention may be considered necessary even without their consent.

\section{Clinical Efficacy of Inhaled Loxapine}

Loxapine is a first-generation antipsychotic with an atypical neurotransmitter receptor affinity profile, as it is much more active at serotonin (5-HT) receptors than at dopamine receptors. It was introduced in psychiatric practice in USA, Canada and Europe 40 years ago, and it is currently approved for the first-line treatment of psychotic symptoms in Canada and as the first choice to treat agitation in France [16, 30]. 
In 2012, inhaled loxapine was approved by the US Food and Drug Administration for the acute treatment of agitation associated with schizophrenia or bipolar I disorder in adults [31]. The European Medicines Agency granted approval for the acute treatment of mild-to-moderate agitation in adults with schizophrenia or bipolar I disorder in 2013 [32]. Inhaled loxapine is delivered by $\mathrm{OBT}^{\circledR}$ (one-breath technology) using the Staccato ${ }^{\circledR}$ system. This is a hand-held, single-use, breath-actuated inhaler that has been specifically designed to rapidly deliver loxapine as a dry powder into the alveoli of the lungs [17]. Inhaled loxapine delivered in this manner exhibits very fast absorption, similar to intravenous administration, which provides a quick onset of effect, and less variability of onset of effect than oral or IM administration. Such rapid and reliable onset of effect is crucial in the time-critical situation of agitation control. Moreover, it provides a reduced level of systemic exposure to patients, which is thought to explain the lower risk of pharmacodynamicrelated adverse effects (extrapyramidal symptoms, vegetative effects) compared with other antipsychotics delivered systemically $[33,34]$.

\subsection{Placebo-Controlled Studies}

The efficacy of inhaled loxapine for the treatment of acute agitation has been evaluated in one phase III randomised controlled trial among patients with schizophrenia [35] and a further phase III study in patients with bipolar disorder [36]. These studies included patients with clinically relevant agitation as measured by a Positive and Negative Syndrome Scale-Excited Component (PANSS-EC) score of at least 14 points and a score of at least 4 points on an individual PANSS-EC item. Patients received a single 4.5- or 9.1-mg dose and were permitted to receive a second dose after $2 \mathrm{~h}$ and a third dose at least $4 \mathrm{~h}$ after dose two if required. The design and main outcomes of these trials are summarised in Table 1.
Patients (adults 18-65 years) in both studies treated with either a single 4.5- or 9.1-mg dose of inhaled loxapine experienced a rapid and robust reduction in agitation symptoms $[35,36]$. In both studies, patients treated with inhaled loxapine achieved statistically significantly greater reductions in the PANSS-EC score compared with patients who received placebo as early as 10 min after dosing, the first assessment point ( $p<0.0001$ for both inhaled loxapine doses vs. placebo in both studies; $[35,36]$. Improvements in elements of the PANSS-EC (tension, excitement, hostility, uncooperativeness and poor impulse control) were noted at the first assessment point (10 min post-dose) and at all subsequent timepoints during $24 \mathrm{~h}$ of follow-up [37]. A post hoc analysis of data from the two phase III studies showed that the proportion of patients achieving a treatment response $(\geq 40 \%$ reduction in the PANSS-EC score) was significantly greater with inhaled loxapine compared with placebo at all timepoints to $2 \mathrm{~h}$ post-dose [38]. The number needed to treatment to achieve $\mathrm{a} \geq 40 \%$ reduction in the PANSS-EC score at $2 \mathrm{~h}$ after delivery of a 10-mg dose was 4 among patients with schizophrenia-associated agitation and 3 for patients with bipolar disorder-associated agitation [39]. Importantly, comparable improvements were noted for patients with higher levels of agitation (PANSS-EC score $>17$ ) and those with lower levels of agitation (PANSS-EC score $\leq 17$ ) at baseline [37]. The majority of patients required a single dose of loxapine and $<10 \%$ of patients required rescue therapy of IM lorazepam (Table 1).

\subsection{Active-Treatment Controlled Study}

Most recently, loxapine has been evaluated in a phase IIIb head-to-head study with IM aripiprazole as a comparator agent $[40,41]$. The PLACID study was performed at 23 centres in the Czech Republic, Germany, Spain and Russia and included 357 adults (aged 18-65 years) with a confirmed diagnosis of schizophrenia or bipolar disorder who presented with acute agitation while hospitalised or while attending an

Table 1 Inhaled loxapine phase III clinical studies: efficacy outcomes

\begin{tabular}{|c|c|c|c|c|c|c|c|c|}
\hline \multirow[t]{2}{*}{ Study } & \multirow[t]{2}{*}{ Treatments } & \multirow[t]{2}{*}{$N$} & \multirow{2}{*}{$\begin{array}{l}\text { PANSS-EC change } \\
\text { from baseline at } 2 \mathrm{~h}\end{array}$} & \multirow{2}{*}{$\begin{array}{l}\% \text { of PANSS-EC } \\
\text { responders }^{\mathrm{a}} \text { at } 2 \mathrm{~h}\end{array}$} & \multicolumn{3}{|c|}{ Number of doses (\%) } & \multirow[t]{2}{*}{$\operatorname{Rescue}^{\mathrm{b}}(\%)$} \\
\hline & & & & & 1 & 2 & 3 & \\
\hline \multirow[t]{3}{*}{ Schizophrenia [35] } & Placebo & 115 & -5.5 & 38.3 & 46.1 & 29.6 & 8.7 & 15.6 \\
\hline & Loxapine $4.5 \mathrm{mg}$ & 116 & $-8.1^{*}$ & 62.9 & 54.4 & 30.7 & 8.8 & 6.1 \\
\hline & Loxapine $9.1 \mathrm{mg}$ & 112 & $-8.6^{* *}$ & 69.6 & 60.9 & 26.4 & 7.3 & 5.4 \\
\hline \multirow[t]{3}{*}{ Bipolar disorder [36] } & Placebo & 105 & -4.9 & 27.6 & 26.7 & 41.0 & 11.4 & 21.0 \\
\hline & Loxapine $4.5 \mathrm{mg}$ & 104 & $-8.1 * *$ & 62.5 & 41.3 & 44.2 & 5.8 & 8.6 \\
\hline & Loxapine $9.1 \mathrm{mg}$ & 10 & $-9.0 * *$ & 73.3 & 61.5 & 26.0 & 3.8 & 8.6 \\
\hline
\end{tabular}

PANSS-EC Positive and Negative Syndrome Scale-Excited Component, $* p<0.01 ; * * p<0.0001$

${ }^{a}$ Defined as a PANSS-EC score change from baseline $\geq 40 \%$

${ }^{\mathrm{b}}$ Intramuscular lorazepam 
emergency room. Patients were randomly assigned (1:1) to inhaled loxapine or IM aripiprazole in an open-label manner with the staff who performed the clinical evaluations blinded to treatment assignment. As for the pivotal phase III trials, a second dose of the study drug was permitted at least $2 \mathrm{~h}$ after the first dose. The primary clinical endpoint in the PLACID trial was the time to treatment response defined as a Clinical Global Impression of Improvement (CGI-I) score of 1 (very much improved) or 2 (much improved).

Among the 357 patients included in the efficacy analysis, 297 had a diagnosis of schizophrenia and 60 had a diagnosis of bipolar I disorder [41]. The majority $(n=236)$ were considered to be moderately and markedly agitated at baseline with nine patients considered to be severely agitated. A total of 179 patients were assigned inhaled loxapine and 178 patients were assigned to IM aripiprazole.

The mean time to CGI-I response was significantly shorter $(p=0.0005)$ among patients treated with inhaled loxapine (median $50 \mathrm{~min}$; 95\% confidence interval 30.0-50.0) than with IM aripiprazole (median $60 \mathrm{~min}$; 95\% confidence interval 50.0-90.0) [41]. When evaluated separately, the time to CGI-I response remained statistically significantly in favour of inhaled loxapine for patients diagnosed with schizophrenia compared with those treated with IM aripiprazole (50 min vs. $60 \mathrm{~min}, p=0.0028$ ), but failed to achieve statistical significance among those with a diagnosis of bipolar disorder ( $30 \mathrm{~min}$ vs. $50 \mathrm{~min}, p=0.06$ ), likely owing to the small sample size of this cohort $(n=60)$. Significantly more patients who were treated with inhaled loxapine achieved a CGI-I response during the first $60 \mathrm{~min}$ of treatment compared with IM aripiprazole (69.8\% vs. $56.2 \%$, respectively; $p=0.0075$ ) (Fig. 3). Moreover, $14 \%$ of patients treated with inhaled loxapine achieved a CGI-I response within $10 \mathrm{~min}$ of dosing compared with $3.9 \%$ of those treated with IM aripiprazole $(p=0.0009)$, and at $20 \mathrm{~min}$, response rates were $29.6 \%$ and $9.0 \%(p<0.0001)$, respectively. Few patients required a second dose of either medication $(6.7 \%$ of those treated with inhaled loxapine and $9.6 \%$ of those treated with IM aripiprazole).

The results of the PLACID study have shown, for the first time, that an inhaled medication-loxapine-is more effective in relieving the symptoms of acute agitation in patients with schizophrenia or bipolar disorder than an IM medication-aripiprazole [41]. The rapid relief of acute agitation in the emergency psychiatric setting is essential to minimise the risks of self-harm and violence to others or to property. The PLACID study employed a pragmatic approach and was conducted in real-world settings, both hospital and emergency room, enrolling patients who would likely receive the medication in routine medical practice [41].

Key exclusion criteria of these studies were agitation primarily because of acute alcohol or drug intoxication/ withdrawal and those considered to be at serious risk for suicide. Nevertheless, real-world evidence showed that acute agitation was effectively and quickly managed by inhaled loxapine in intoxicated patients in a retrospective review of data from a case series $[42,43]$.

\section{Safety of Inhaled Loxapine}

\subsection{Placebo-Controlled Studies}

In the pooled phase II and III clinical evaluations of inhaled loxapine (excluding the PLACID study), the most frequently reported adverse events with an incidence $\geq 2 \%$ than with placebo were taste distortion (dysgeusia), sedation and throat irritation $[31,32,37]$. Dysgeusia was reported by $11.3 \%$ of patients treated with inhaled loxapine $4.5 \mathrm{mg}, 14.3 \%$ of those treated with inhaled loxapine $9.1 \mathrm{mg}$ and $4.9 \%$ of patients who received placebo. Rates of sedation/somnolence were $12.1,12.0$ and $9.5 \%$, respectively, and rates for throat irritation were $0.8,2.7$ and $0.4 \%$, respectively $[31,32,37]$. Extrapyramidal symptoms emerged for $1.9,1.5$ and $0.4 \%$ of patients, respectively $[31,32,37]$.

The most common airway-related adverse event in the clinical trial programme was cough and occurred in $1.7 \%$ of patients. All but one of these events were regarded as mild in intensity, the additional event being rated as moderate in intensity, and all were self-limiting [37]. Although not observed in the phase III clinical trials, the product label for inhaled loxapine contains a warning for bronchospasm and the potential for subsequent respiratory distress and respiratory arrest $[31,32,37]$. The safety and tolerability of inhaled loxapine with regard to respiratory adverse events have been evaluated in two randomised controlled trials, one in patients with asthma $(n=52)$ and a second in patients with chronic obstructive pulmonary disease (COPD; $n=53$ ) [44]. The studies compared two doses of inhaled loxapine $9.1 \mathrm{mg}$ (delivered $10 \mathrm{~h}$ apart) with placebo. Rescue bronchodilators were withheld for $6-8 \mathrm{~h}$ prior to the first dose to $34 \mathrm{~h}$ after the last dose, unless clinically indicated as rescue medication. Symptomatic bronchospasm was reported for $53.8 \%$ of patients treated with inhaled loxapine in the asthma study and $19.2 \%$ of inhaled loxapine-treated patients in the COPD study. Equivalent rates for placebo were $11.5 \%$ and $11.1 \%$, respectively. The majority of events responded to rescue bronchodilator therapy within $1 \mathrm{~h}$ with all events resolving at the time of the last spirometry evaluation. None of the events were regarded as serious [44]. Inhaled loxapine is contraindicated for patients with a history of asthma, COPD or other lung diseases associated with bronchospasm, those with acute respiratory signs/symptoms and those currently receiving medications to treat airway disease $[31,32$, 37]. The safety and efficacy of inhaled loxapine in patients 
aged $>65$ years or in elderly patients, including those with dementia-related psychosis, have not been established.

\subsection{Active-Treatment Controlled Study}

In the PLACID study, both treatments were well tolerated, and no new safety signals were identified for inhaled loxapine beyond those observed in the placebo-controlled clinical trials [41]. The most commonly reported adverse events were dizziness $(2.2 \%$ with inhaled loxapine and $6.2 \%$ with IM aripiprazole), dysgeusia (12.3\% with inhaled loxapine and no patient treated with IM aripiprazole) and somnolence (14.5\% with inhaled loxapine and $14.1 \%$ with IM aripiprazole) [37]. Although, per-protocol patients with active airway disease should have been excluded from participation, four patients with asthma were included and two were randomised to inhaled loxapine. No bronchospasm events were reported for these patients [41].

\section{Patient Satisfaction}

Ensuring patients are satisfied with the treatment they receive for their acute episode of agitation has been recognised as an important component of the profile of an 'ideal' antipsychotic [7]. One of the reasons patients do not attend for professional help until late in the escalation continuum (i.e. with more severe agitation symptoms), is previous "bad"/traumatic experiences as a result of the use of coercive measures. Avoiding physical restraint or the traumatic delivery of medication may contribute to patient satisfaction with treatment and increase the likelihood that they will seek and cooperate with medical assistance for future episodes of agitation [45].

Patient satisfaction was measured as part of the PLACID study using the Treatment Satisfaction Questionnaire for Medication, specifically the patient's response to item 14 "Taking all into account, how satisfied or dissatisfied do you feel with the treatment?". Statistically significantly more patients treated with inhaled loxapine indicated they were 'very' or 'extremely' satisfied with their treatment than among those who were treated with IM aripiprazole (53.3\% vs. $36.4 \%$; $p=0.0012)[41]$.

\section{Cost of Managing Inpatient Agitation}

There are limited data available to assess the costs of agitation associated with schizophrenia or bipolar disorder, either in the psychiatric inpatient setting or within the community setting. Agitation has been shown to be associated with prolonged hospital admissions, increased readmission rates and increased medication consumption [46, 47]. A UK-based study estimated the cost of agitated behaviour within the inpatient psychiatric care setting to be $£ 178.6$ million in 2005 for containment and intervention [48]. The cost of time spent by healthcare professionals on containment measures for acutely agitated psychiatric inpatients in Spain in 2014 was estimated to be $€ 27$ million [49].

A systematic evaluation of the costs of psychiatric inpatient agitation in the South Barcelona region of Spain has recently been reported [50]. The study determined the number of agitation events during 2013 and the cost of each individual intervention. The study captured data for 808 patients admitted for acute inpatient care during 2013, with a total of 918 admissions. These admissions accounted for 22,550 patient bed days with a median length of stay of 21.79 days. During these admissions, 245 episodes of restraint and 155 episodes of seclusion were recorded. The total cost of interventions was $€ 280,535$ with the lowest costs associated with verbal containment plus surveillance $(€ 36,150)$ and higher and almost equivalent costs associated with verbal containment plus surveillance plus seclusion $(€ 120,835)$ and verbal containment plus surveillance plus seclusion plus restraint $(€ 121,881)$. Costs (medication, staff hours and other resources) were markedly higher when patients exhibited aggression against objects as this was the most frequent level of agitation seen in this study (Table 2). The results of the study suggest that early intervention with the aim of preventing escalation to aggression may reduce the need for seclusion and restraint and thus reduce accrued costs of managing agitation in the acute psychiatric inpatient setting.

\section{Managing Agitation in Community-Dwelling Patients with Schizophrenia or Bipolar Disorder}

To date, the study of agitation in patients with schizophrenia and bipolar disorder, both from a phenomenological perspective and with regard to management, has focused on the acute care setting-either the emergency room or inpatient hospital. Patients experience initial symptoms and escalate unpredictably to a more severe level of agitation in outpatient settings such as their homes or workplaces, where early and rapid symptom relief is needed to avoid the need for emergency room attendance or psychiatric hospitalisation. Indeed, agitation places a considerable burden both on community-dwelling patients and their caregivers as has been discussed [51, 52].

The impact of agitation on community-dwelling adults with schizophrenia or bipolar disorder has been evaluated in a survey of 585 patients in Germany, Spain and the UK conducted between October 2016 and January 2017 [52]. The survey captured information about their demographics, their 
Table 2 Unitary costs of each intervention according to the agitation state (based on [50]

\begin{tabular}{|c|c|c|c|c|c|c|}
\hline & AAI & MA & AAO & AAP & & $>300$ \\
\hline L1 & 282,34 & 326,53 & 422,43 & 397,83 & & $300-400$ \\
\hline L2 & 391,86 & 430,71 & 437,61 & 533,81 & & $400-500$ \\
\hline L3 & - & - & 788,61 & 821,99 & & $500-600$ \\
\hline & & & & & & $>600$ \\
\hline
\end{tabular}

$A A I$ anxiety and irritability, $A A O$ agitation with aggression against objects, $A A P$ agitation with aggression against persons, $L 1$ verbal containment plus surveillance, $L 2$ verbal containment plus surveillance plus seclusion, $L 3$ verbal containment plus surveillance plus seclusion plus restraint, $M A$ moderate agitation

agitation episodes and how episodes of agitation impact their daily life and revealed that patients experience frequent episodes of agitation ranging in intensity from mild to severe. Typical symptoms included feeling uneasy, restless, nervous or tense (Fig. 1). On average, the cohort reported experiencing 22.4 episodes of mild agitation, 15.4 episodes of moderate agitation, 6.8 episodes of moderate-to-intense agitation and 2.9 episodes of severe agitation in the preceding 12 months. Hospital visits were common with half of the patients reporting that they had attended hospital because of episodes of agitation in the preceding 12 months. Despite the majority of patients $(71 \%)$ confirming that they were always or sometimes aware they were becoming agitated (Fig. 2), patients typically waited more than a week before going to hospital to seek medical care. During this time, they reported attempting a variety of coping strategies to relieve their agitation. The most common strategies included taking prescribed medication (55\% of patients with schizophrenia, $66 \%$ of patients with bipolar disorder), speaking to friends or family (53\% and $60 \%$, respectively), speaking to a healthcare professional ( $52 \%$ and $62 \%$, respectively), requesting rescue medication ( $46 \%$ and $40 \%$, respectively), doing something relaxing ( $43 \%$ and $53 \%$, respectively) or removing themselves from a stressful situation $(36 \%$ and $53 \%$, respectively). However, $16 \%$ of patients stated that nothing was able to relieve their agitation and $56 \%$ reported being only sometimes able to control their agitation (Fig. 3).

These data highlight, for the first time, the substantial burden experienced by community-dwelling patients with schizophrenia or bipolar disorder as a result of agitation and that violence and aggressiveness—often the reason for stigmatisation of such patients - are only present in a minority of cases. The delay between patients experiencing agitation and attending hospital is of particular concern as it suggests that by the time they attend for medical care their symptoms have persisted for some time and may already have escalated. Additionally, of note is the observation that speaking to a caregiver or healthcare professional successfully helped some patients cope with their agitation. However, of the $56.5 \%$ of patients who reported speaking to friends or family, only $39 \%$ reported that this was a successful strategy for them. Similarly, of the $57 \%$ of patients who reported speaking to a healthcare professional, only $38.5 \%$ reported

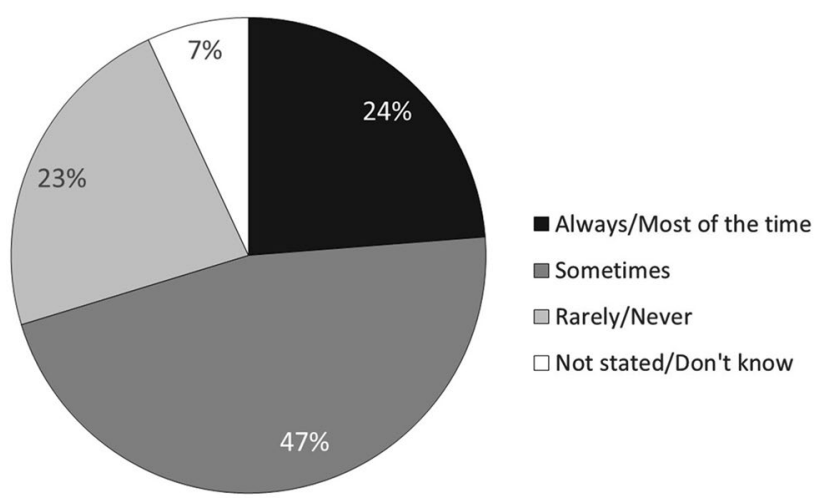

Fig. 2 Self-reported patient awareness of when they are becoming agitated [52]
Fig. 1 Symptoms associated with agitation reported by community-dwelling adults with bipolar disorder or schizophrenia [52]

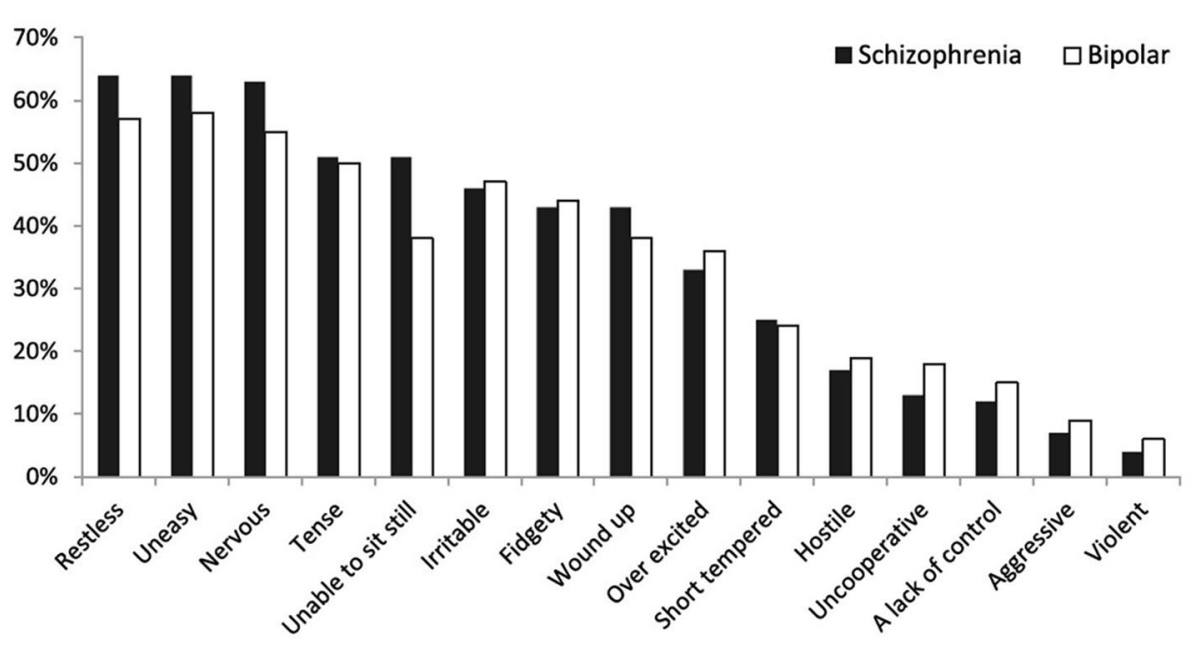


that this was a successful strategy for them. These observations highlight that, at least in the community setting, talking therapies are insufficient to fully resolve episodes of agitation for the majority of patients.

Alongside the patient-focused survey described above, a survey of caregivers was also conducted [51]. A total of 297 caregivers of patients who took part in the patient survey completed the caregiver survey. Caregivers were mainly family members, with $39 \%$ being the partner or spouse of the patient, $18 \%$ being the parent, $14 \%$ being a sibling and $13 \%$ being a child of the patient. The majority were female (72\%) and were working full time $(32 \%)$ or part time $(32 \%)$. On average, caregivers provided $38.3 \mathrm{~h}$ of care each week with $20 \%$ providing $50 \mathrm{~h}$ of care or more. Caregivers reported being able to recognise a variety of symptoms during an episode of agitation including when the patient felt tense $(65 \%)$, restless $(64 \%)$, uneasy $(63 \%)$, nervous $(68 \%)$ or irritable (54\%). The burden of their caregiving activities was captured using the Caregiving Consequences of Psychiatric Disorders component of the Involvement Evaluation Questionnaire [53]. Higher scores on this validated 31-item tool indicate greater caregiver burden. The mean Involvement Evaluation Questionnaire score for the total cohort was 32.2 (standard deviation 15.27) and while caregiver burden was similar for those caring for patients with schizophrenia and those caring for patients with bipolar disorder, symptoms of hostility and a lack of control during an episode of agitation were associated with higher Involvement Evaluation Questionnaire scores.

A recent case series highlighted the potential clinical utility of inhaled loxapine for the treatment of agitation outside the hospital setting [54]. The cohort consisted of 14 community-dwelling adults with a diagnosis of a psychiatric disorder (schizophrenia, bipolar disorder or schizoaffective disorder) presenting for pre-hospital emergency care between December 2015 and November 2016 [54]. All 14 patients presented with agitation that required immediate treatment and 12 of the 14 patients consented to receive inhaled loxapine. Of these, two patients were assessed as having mild agitation, three as having moderate agitation, two as having moderate-to-severe agitation, four as having severe agitation and one as having extreme agitation. All patients experienced symptomatic relief between 2 and 10 min after dosing. Two patients required additional therapy with intranasal midazolam, the administration of which was achieved in a non-coercive manner. The two patients who refused inhaled loxapine were both assessed as having extreme agitation that likely compromised their ability to make rational care decisions.

A study is now underway to further evaluate the usefulness of inhaled loxapine outside the hospital setting (NCT02525991). This phase IV study will evaluate the safety of self-administered inhaled loxapine in patients with schizophrenia or bipolar disorder outside the hospital setting and without the direct supervision of a healthcare professional. The initial results for the first ten patients (five with schizophrenia and five with bipolar disorder) of the study are promising; 10 min after the use of inhaled loxapine, six patients assessed the agitation as "Very much improved", one patient as "Much improved", and three patients as "Minimally improved" [55].

As observed in the survey of community-dwelling patients with schizophrenia or bipolar disorder described above [52], self-administration of prescribed medication is a common coping strategy adopted by patients (reported by $60 \%$ of patients) but is reported as being successful by only $47.5 \%$ of patients [52]. These data highlight the need for more effective medications for self-administration to address the frequent episodes of agitation experienced by community-dwelling patients with schizophrenia or bipolar disorder. The survey also revealed that patients typically wait a week before presenting for medical care for episodes of
Fig. 3 PLACID study cumulative responder analysis [41]. CGI-I Clinical Global Impression of Improvement, $I M$ intramuscular, ${ }^{*} p<0.05 ; * * p<0.01$; $* * * p<0.0001$

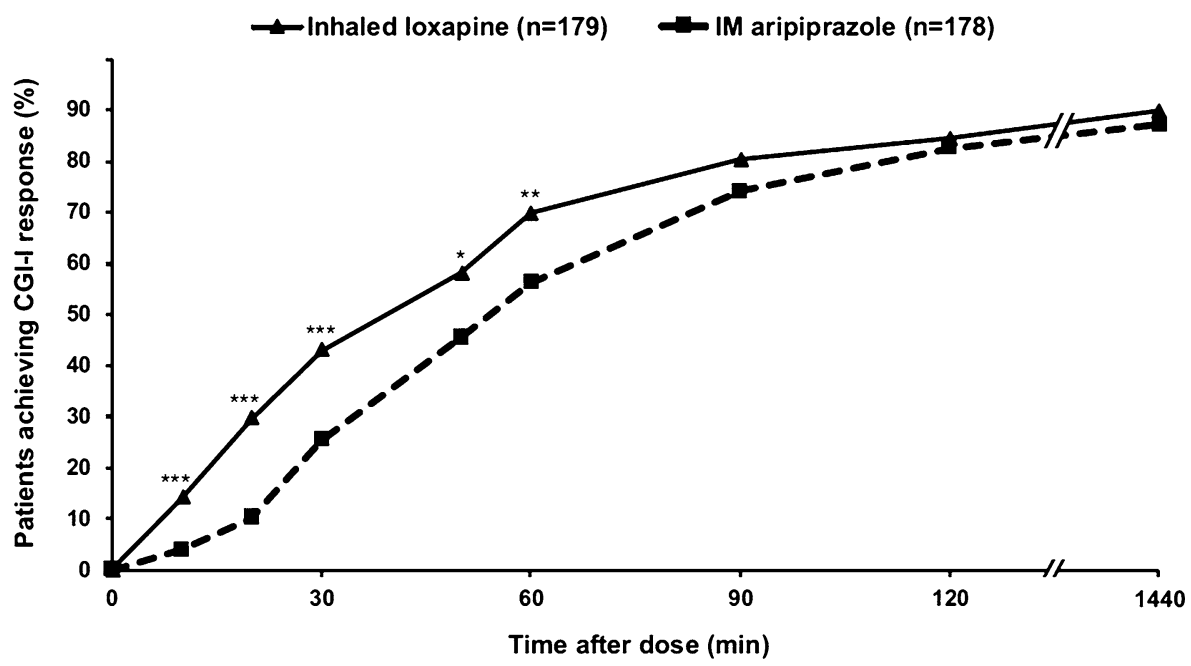


agitation, by which time their symptoms may have escalated necessitating a hospital stay.

\section{Expert Commentary}

A number of factors are driving the current evolution of the management strategy for agitation in patients with schizophrenia or bipolar disorder. Among these are the recognition of the need to minimise the use of coercive and traumatic interventions, including mechanical physical restraint, owing to its negative impact on the therapeutic alliance between patients and their healthcare providers, and the availability of medications that can be taken orally or via inhalation. Rapid relief from the symptoms of agitation is an important component of managing patients experiencing acute agitation and, while less traumatic than an IM injection, patients must endure a delay between the administration of an oral medication and the onset of the therapeutic effect. The availability of inhaled loxapine has been shown to provide rapid symptom relief that can be perceived by patients within 10 min of administration $[35,36]$.

In addition, the new non-traumatic route of administration, a rapid delivery system and a lack of sedative effects make inhaled loxapine not only an ideal treatment for patients who are cooperative and remain non-aggressive [12] but also a truly ethical treatment option. The offer of a self-administered inhaled drug such as loxapine in the early stages of agitation may be a significant step towards the establishment of a solid therapeutic alliance and, at the same time, it may help to identify actually cooperative patients. Inhaled loxapine can be offered in almost all cases of agitation as part of the initial de-escalation. All cooperative agitated patients can easily self-administer the inhaled drug. As administration requires only a single breath without the need to coordinate the device, even patients who are more severely functionally impaired can be assisted to take the medication provided they are cooperative and non-aggressive. Verbal de-escalation and support can be continued with the patient remaining able to communicate with their healthcare provider and express his/her feelings and wishes as the episode resolves. By integrating inhaled loxapine into protocols for the management of acute agitation that emphasise verbal de-escalation and non-traumatic management strategies, the therapeutic alliance can remain intact and patients will feel confident to reutilise the inhaled medication.

Effective deployment of inhaled loxapine in an acute setting requires that the medication is immediately available to the healthcare provider once the agitation is recognised, and delivered as part of a protocol involving environmental and verbal management, with the support of additional team members who may intervene if patient safety or the safety of others is compromised. Given the potential for a bronchospasm in patients with established respiratory conditions such as asthma and COPD, rescue medication such as salbutamol should also be available.

The ease of delivery and rapid onset of action of inhaled loxapine suggest a potential for use outside the acute emergency or hospital setting by community-dwelling patients with schizophrenia or bipolar disorder. The opportunity for community-dwelling patients to intervene early in the course of an episode of agitation with an effective self-administered agent may not only reduce the time spent in an agitated state but also reduce or avoid agitation-related hospitalisations and associated costs.

\section{Conclusions}

This is an interesting time for the treatment of agitated patients. A combination of factors is changing the way agitated patients are treated, bringing the quality of care to a new level. These factors include the use of innovative methods and treatments (such as inhaled loxapine), the change in professional habits (patient-professional shared decision-making process, therapeutic alliance, patient-centric approach) and the change in disease awareness (de-stigmatisation, patient empowerment and reduction in the use of traumatic management options).

The advancement in the management of the agitated patient is evolving rapidly and this is reflected in recently updated clinical guidelines. It is now time for the medical community to adapt to the new standard of care, and promote education and training among the therapeutic teams, patients and caregivers.

Engaging competent patients in decision making regarding their treatment, should preserve and improve the therapeutic alliance with healthcare professionals and patient compliance with their treatment, improving the experience of all parties involved.

Acknowledgements The authors thank Dr. Alberto Moldón of Adelphi Spain for help with writing the manuscript.

\section{Compliance with Ethical Standards}

Funding No funding was received for the preparation of this article.

Conflict of interest Bruno Pacciardi has no conflicts of interest that are directly relevant to the content of this article. Alfredo Calcedo has received honoraria for consulting work from Ferrer International. Thomas Messer has received honoraria for consulting work and clinical speeches from Ferrer International. Ferrer International was given the opportunity to check the data used in this manuscript for factual accuracy only.

Open Access This article is distributed under the terms of the Creative Commons Attribution-NonCommercial 4.0 International License 
(http://creativecommons.org/licenses/by-nc/4.0/), which permits any noncommercial use, distribution, and reproduction in any medium, provided you give appropriate credit to the original author(s) and the source, provide a link to the Creative Commons license, and indicate if changes were made.

\section{References}

1. World Health Organization. Mental disorders. http://www.who. int/en/news-room/fact-sheets/detail/mental-disorders. Accessed Jun 2018.

2. Rybakowski JK. Recent advances in the understanding and management of bipolar disorder in adults. F1000Res. 2017;6:2033.

3. Tondo L, et al. Depression and mania in bipolar disorder. Curr Neuropharmacol. 2017;15:353-8.

4. Duarte $\mathrm{W}$, et al. The relationship between neurocognitive functioning and occupational functioning in bipolar disorder: a literature review. Eur J Psychol. 2016;12:659-78.

5. Piotrowski P, et al. Causes of mortality in schizophrenia: a updated review of European studies. Psychiat Danub. 2017;29:108-20.

6. Tripathi A, et al. Cognitive deficits in schizophrenia: understanding the biological correlates and remediation strategies. Clin Psychopharmacol Neurosci. 2018;16:7-17.

7. Garriga M, et al. Assessment and management of agitation in psychiatry: expert consensus. World J Biol Psychiatry. 2016;17:86-128.

8. Sachs GS. A review of agitation in mental illness: burden of illness and underlying pathology. J Clin Psychiatry. 2006;67(Suppl. 10):5-12.

9. American Psychiatric Association. Diagnostic and statistical manual of mental disorders. 4th ed. Washington, DC: American Psychiatric Association; 2000.

10. Patel MX, et al. Joint BAP NAPICU evidence-based consensus guidelines for the clinical management of acute disturbance: de-escalation and rapid tranquillisation. J Psychopharmacol. 2018;32:601-40.

11. Hankin CS, et al. Agitation in the inpatient psychiatric setting: a review of clinical presentation, burden, and treatment. J Psychiatr Pract. 2011;17:170-85.

12. Martinez-Raga J, et al. 1st International Experts' Meeting on Agitation: conclusions regarding the current and ideal management paradigm of agitation. Front Psychiatry. 2018;9:54.

13. Allen MH, Currier GW, Hughes DH, Expert Consensus Panel for Behavioral Emergencies, et al. The expert consensus guideline series: treatment of behavioural emergencies. Postgrad Med. 2001;(Spec No):1-88.

14. Jorgensen TR, et al. The effect of the medicine administration route on health-related quality of life: results from a time tradeoff survey in patients with bipolar disorder or schizophrenia in 2 Nordic countries. BMC Psychiatry. 2016;16:244.

15. Jorgensen TR, et al. Patient preferences for medicine administration for acute agitation: results from an internet-based survey of patients diagnosed with bipolar disorder or schizophrenia in two Nordic countries. Psychol Health Med. 2018;23:30-8.

16. Popovic D, et al. Revisiting loxapine: a systematic review. Ann Gen Psychiatry. 2015;14:15.

17. Dinh K, et al. In vitro aerosol characterization of Staccato loxapine. Int J Pharm. 2011;403:101-8.

18. Marco CA, Vaughan J. Emergency management of agitation in schizophrenia. Am J Emerg Med. 2005;23:767-76.

19. San L, et al. State of acute agitation at psychiatric emergencies in Europe: the STAGE study. Clin Pract Epidemiol Ment Health. 2016;12:75-86.
20. Buckley PF, et al. Treatment of the psychotic patients who is violent. Psychiatr Clin North Am. 2003;26:231-72.

21. Stahl SM, et al. "Meta-guidelines" for the management of patients with schizophrenia. CNS Spectr. 2013;18:150-62.

22. Fountoulakis KN, et al. The International College of Neuro-Psychopharmacology (CINP) treatment guidelines for bipolar disorder in adults (CINP-BD-2017). Part 3. The clinical guidelines. Int J Neuropsychopharmacol. 2017;20:180-95.

23. Harrison PJ, et al. The emerging neurobiology of bipolar disorder. Trends Neurosci. 2018;41:18-30.

24. Lehman AF et al. Practice guideline for the treatment of patients with schizophrenia. 2nd ed. 2004. https://psychiatryonline.org/pb/ assets/raw/sitewide/practice_guidelines/guidelines/schizophrenia. pdf. Accessed Jun 2018.

25. Mielau J, et al. Subjective experience of coercion in psychiatric care: a study comparing the attitudes of patients with healthy volunteers towards coercive methods and their justification. Eur Arch Psychiatry Clin Neurosci. 2016;266:337-47.

26. Mohr WK, et al. A restraint on restraints: the need to reconsider the use of restrictive interventions. Arch Psychiatr Nurs. 1998;12:95-106.

27. Bundesverfassungsgericht. Press release no. 62/2018. Constitutional requirements regarding the use of physical restraints on patients confined under public law in psychiatric hospitals. 2018. https://www.bundesverfassungsgericht.de/SharedDocs/Pressemitt eilungen/EN/2018/bvg18-062.html. Accessed 7 Sep 2018.

28. Vieta E, et al. Protocol for the management of psychiatric patients with psychomotor agitation. BMC Psychiatry. 2017;17:328.

29. United Nations General Assembly. Session 61 Resolution 106. Convention on the rights of persons with disabilities. A/ RES/61/106; 2006.

30. Horn M, et al. Prise en charge médicamenteuse des agitations aux urgences: recommandations théoriques et études des pratiques. Presse Med. 2015;44(1):20-6.

31. US Food and Drug Administration. Adasuve ${ }^{\circledR}$ [prescribing information]. 2017. http://adasuve.com/PDF/AdasuvePI.pdf. Accessed Jun 2018.

32. European Medicines Agency. Adasuve ${ }^{\circledR} 9.1 \mathrm{mg}$ inhalation powder. SmPC. https://www.medicines.org.uk/emc/medicine/32246. Accessed Jun 2018.

33. Spyker DA, et al. Pharmacokinetics of loxapine following inhalation of a thermally generated aerosol in healthy volunteers. J Clin Pharmacol. 2010;50(2):169-79.

34. Spyker DA, et al. Multiple dose pharmacokinetics of inhaled loxapine in subjects on chronic, stable antipsychotic regimens. J Clin Pharmacol. 2015;55(9):985-94.

35. Lesem MD, et al. Rapid acute treatment of agitation in individuals with schizophrenia: multicentre, randomised, placebo-controlled study of inhaled loxapine. Br J Psychiatry. 2011;198:1-58.

36. Kwentis J, et al. Rapid acute treatment of agitation in patients with bipolar I disorder: a multicenter, randomized, placebocontrolled clinical trial with inhaled loxapine. Bipolar Disord. 2012;14:31-40.

37. Citrome L. Addressing the need for rapid treatment of agitation in schizophrenia and bipolar disorder: focus on inhaled loxapine as an alternative to injectable agents. Ther Clin Risk Manag. 2013;9:235-45.

38. Zeller S, et al. Response to inhaled loxapine in patients with schizophrenia or bipolar I disorder: PANSS-EC responder analyses. $\mathrm{Br}$ J Psych Open. 2017;3:285-90.

39. Citrome L. Inhaled loxapine for agitation revisited: focus on effect sizes from two phase III randomised controlled trials in persons with schizophrenia or bipolar disorder. Int J Clin Pract. 2012;66:318-25.

40. San L, et al. Rationale and design of the PLACID study: a randomised trial comparing the efficacy and safety of inhaled 
loxapine versus IM aripiprazole in acutely agitated patients with schizophrenia or bipolar disorder. BMC Psychiatry. 2017;17:126.

41. San L, et al. PLACID study: a randomized trial comparing the efficacy and safety of inhaled loxapine versus intramuscular aripiprazole in acutely agitated patients with schizophrenia or bipolar disorder. Eur Neuropsychopharmacol. 2018;18:30073-7.

42. Roncero C, et al. Effectiveness of inhaled loxapine in dualdiagnosis patients: a case series. Clin Neuropharmacol. 2016;39(4):206-9.

43. Roncero $\mathrm{C}$, et al. Inhaled loxapine for agitation in intoxicated patients: a case series. Clin Neuropharmacol. 2017;40(6):281-5.

44. Gross N, et al. Safety and tolerability of inhaled loxapine in subjects with asthma and chronic obstructive pulmonary disease: two randomized controlled trials. J Aerosol Med Pulm Drug Dev. 2014;27:478-87.

45. Richmond JS, et al. Verbal de-escalation of the agitated patient: consensus statement of the American Association for Emergency Psychiatry Project BETA De-escalation Workgroup. West J Emerg Med. 2012;13:17-25.

46. Cots F, et al. Hospital costs associated with agitation in the acute care setting. Psychiatr Serv. 2016;67:124-7.

47. Rubio-Valera M, et al. Health service use and costs associated with aggressiveness or agitation and containment in adult psychiatric care: a systematic review of the evidence. BMC Psychiatry. 2015;15:35.

48. Flood C, et al. Estimating the costs of conflict and containment on adult acute inpatient psychiatric wards. Nurs Econ. 2008;26:325-30.

49. Garrido E, et al. El coste económico de los procedimientos de contención mecánica de origen psiquiátrico en España. Psiquiatr Biol. 2015;22:12-6.
50. Serrano-Blanco A, et al. In-patient costs of agitation and containment in a mental health catchment area. BMC Psychiatry. 2017;17:212.

51. Blanthorn-Hazell S, et al. A survey of caregiver burden in those providing informal care for patients with schizophrenia or bipolar disorder with agitation: results from a European study. Ann Gen Psychiatry. 2018;17:8.

52. Roberts $\mathbf{J}$, et al. Characterizing the experience of agitation in patients with bipolar disorder and schizophrenia. BMC Psychiatry. 2018;18:104.

53. van Wijngaarden B, et al. Caregiving in schizophrenia: development, internal consistency and reliability of the Involvement Evaluation Questionnaire-European version. EPSILON Study 4. European psychiatric services: inputs linked to outcome domains and needs. Br J Psychiatry. 2000;39:s21-7.

54. Cester-Martinez A, et al. New medical approach to out-of-hospital treatment of psychomotor agitation in psychiatric patients: a report of 14 cases [in Spanish]. Emergencias. 2017;29:182-4.

55. Gil E, Garcia-Alonso F, Boldeanu A, et al. Safety and efficacy of self-administered inhaled loxapine (Adasuve ${ }^{\circledR}$ ) in agitated patients outside the hospital setting: protocol for a phase IV, single-arm, open-label trial. BMJ Open. 2018;8(10):e020242.

56. Cousin FR, et al. Therapeutics approach of adult patients with acute and chronic psychotic states: REALITE survey [in French]. Encéphale. 2006;32:466-73.

57. Currier GW, Allen MH. Emergency psychiatry: physical and chemical restraint in the psychiatric emergency service. Psychiatr Serv. 2000;51:717-9.

58. De Hert M, et al. The prevention of deep vein thrombosis in physically restrained patients with schizophrenia. Int J Clin Pract. 2010;64:1109-15. 